\title{
Análisis de las competencias digitales del profesorado universitario desde el modelo TPACK (conocimiento tecnológico y pedagógico del contenido)
}

\section{Analysis of the digital competences of university teachers from the TPACK model (technological and pedagogical knowledge of content)}

\author{
Byron Geovanny Hidalgo Cajo \\ http://orcid.org/0000-0002-5526-1676 \\ Universitat de Lleida, Departament de Tecnología Educativa, Spain \\ Universidad Nacional de Chimborazo, Ecuador \\ Mercé Gisbert Cervera \\ (D) https://orcid.org/0000-0002-8330-1495 \\ Universitat Rovira I Virgili,Departament de Tecnología Educativa, Spain
}

Autor para correspondencia: bhidalgo@unach.edu.ec; merce.gisbert@urv.cat

Fecha de recepción: 24 de agosto de 2020 - Fecha de aceptación: 10 de diciembre de 2020

\section{Resumen}

La formación del profesorado universitario en tecnologías digitales (TD) representa un valor importante en el desarrollo de su labor académica, desde esta perspectiva es necesario analizar profundamente las competencias que posee el profesorado mediante la aplicación del modelo TPACK (Tecnological, Pedagogical, Content Knowledge). Para el análisis del modelo se aplicó un instrumento ya elaborado que fue modificado y se comprobó su validez mediante la revisión de expertos en la rama, de la misma manera se realizó el análisis de consistencia interna mediante el alfa de Cronbach obteniendo un valor de 0.733 . La muestra analizada fue de 238 docentes, el estudio tiene un enfoque cuantitativa, descriptiva y transversal, los principales resultados dan lugar a que las competencias que los docentes poseen están en constante evolución, es así que entre las competencias tecnológicas, pedagógicas y disciplinares de manera independiente logran alcanzar competencias altas en el profesorado, no siendo así las competencias emergentes del TPACK donde se manifiesta una disminución en la integración de las mismas y este efecto posiblemente impacte significativamente en el proceso enseñanza aprendizaje, esto no solo permite conocer la situación actual del profesorado en la integración de las TD, sino también brinda una visión muy general de la formación que los estudiantes adquieren mediados por los entornos digitales. Si bien las competencias tecnológicas que el profesorado desarrolle no es la solución en si a todos los problemas de la educación, se deberá también hacer hincapié que estas competencias se enmarquen en sinergia con los conocimientos pedagógicos y de contenidos, con el fin de lograr la calidad educativa.

Palabras claves: tecnologías digitales; TPACK; competencias docentes; procesos de enseñanza aprendizaje; pedagogía. 


\begin{abstract}
The training of university teachers in digital technologies (TP) represents an important value in the development of their academic work, from that perspective is necessary to analyze in depth the skills that teachers have through the application of the TPACK model (Technological, Pedagogical, Content Knowledge). For the analysis of the model, an already elaborated instrument was applied, which was modified and its validity was verified by means of the review of experts in the branch. In the same way, the analysis of internal consistency was carried out by means of Cronbach's alpha, obtaining a value of 0.733 . The analyzed sample was of 238 teachers, the study has a quantitative, descriptive and transversal approach, the main results give place to the fact that the competences that teachers possess are in constant evolution, so among the technological, pedagogical and disciplinary competences in an independent way they manage to reach high competences in the teaching staff, not being the emerging competences of TPACK where a decrease in their integration is manifested and this effect may impact significantly in the teaching-learning process, this not only allows to know the current situation of teachers in the integration of TD, but also provides a very general vision of the training that students acquire mediated by digital environments. Although the technological skills that teachers develop are not the solution in itself to all educational problems, it should also be emphasized that these skills are framed in synergy with pedagogical and content knowledge, in order to achieve educational quality.

Keywords: TD; TPACK; teaching skills; teaching-learning processes; pedagogical.
\end{abstract}

\title{
Introducción
}

La importancia que están adquiriendo las tecnologías de la información y comunicación (TIC) para la creación de nuevos escenarios hace que la "competencia digital" sea necesaria para que los individuos puedan desenvolverse en la sociedad actual y futura. Tal competencia digital debe entenderse no solo como el simple dominio instrumental, sino como la construcción, producción, evaluación y selección de recursos digitales.

Las competencias digitales en la actualidad ha cobrado un fuerte protagonismo en la educación, es así que ha sido considerado como una de las ocho competencias clave para el aprendizaje permanente por parte del parlamento europeo (Comisión de las Comunidades Europeas, 2006), las mismas que no solo proporciona la capacidad de aprovechar las TD y los retos que plantean, sino que son necesarias para formar parte de la nueva sociedad del siglo XXI.

El uso de la tecnología representa una forma de innovar el proceso enseñanzaaprendizaje aportando aspectos positivos al estudiante y a su conocimiento, es así que la Organización Mundial de la Salud (OMS), considera al uso de las TIC como "herramientas que facilitan la comunicación y el proceso de transmisión de información por medios electrónicos, con el propósito de mejorar el bienestar de los individuos" (OPS-OMS, 2019).

"Las TIC pueden contribuir al acceso universal a la educación, a la equidad en este ámbito, a la calidad del aprendizaje y de la enseñanza, el desarrollo profesional de los docentes, así como también en la mejora de la gestión de la educación, su gobernanza y administración a través de la combinación adecuada de políticas, tecnologías y capacidades” (UNESCO, 2016). 
"Utilizar e incorporar adecuadamente las TD en actividades de enseñanza/aprendizaje es una competencia genérica, común/transversal en cualquier plan de formación de docentes, sin embargo, es una de las competencias que el docente, independientemente de la especialidad que curse, reconoce como no adquirida" (Pérez M., 2008, p. 343-367).

"La competencia digital se basa en saber utilizar e incorporar adecuadamente en las actividades de enseñanza/aprendizaje las tecnologías digitales, por lo cual la formación docente no puede reducirse a la adquisición de competencias digitales o destrezas tecnológicas per se, sino que debe basarse en su aplicación didáctica (Tejada, 2009, p. 9-15)”.

Desde esta perspectiva, es necesario que la formación del profesorado universitario se enfoque en desarrollar competencias necesarias para la utilización de recursos tecnológicos, que ayuden y motiven el aprendizaje en los educandos. Es esencial para la integración de la tecnología en el aula las competencias que el profesorado posea, sin embargo también es de suma importancia el dominio pedagógico y de contenido que transforme los enfoques centrados en el profesor hacia un enfoque centrado en el estudiante (Hidalgo et al., 2019).

La introducción de las TD en las aulas pone en evidencia la necesidad de una nueva definición de roles entre alumnos y docentes, gracias a la aplicación de estas herramientas, los alumnos pueden adquirir mayor autonomía y responsabilidad en el proceso de aprendizaje, lo que obliga al docente a salir de su rol clásico como única fuente de conocimiento, lo que genera incertidumbre, tensión o temor en los docentes, pues se muestran reacios al cambio frente a una realidad que obliga a una reestructuración académica y educativa (UNESCO, 2016).

La principal motivación del estudio radica en la preocupación imperante de conocer las competencias digitales que el profesorado universitario posee en el proceso enseñanza aprendizaje y su influencia en la aplicación y desarrollo de nuevas metodologías de enseñanza basadas en la tecnología moderna. Esto conlleva a que el profesorado reconozca las ventajas que traería el uso de las TD, en el camino a la innovación docente y tecnológica en el proceso educativo.

En este contexto el objetivo de la investigación se enmarca fundamentalmente en analizar e identificar los ámbitos competenciales del profesorado universitario desde el modelo TPACK (conocimiento tecnológico y pedagógico del contenido), de tal manera que sirva como punto de referencia diagnóstica para la formación continua del profesorado con respecto a su competencia digital.

\section{Problemática}

Las instituciones educativas, especialmente las de educación superior, se encuentran en un constante proceso de cambio, buscando siempre la excelencia académica, para lo cual es imperioso contar con un personal docente que sea competente y experto en cada una de las áreas de especialización, además requiere contar con herramientas tecnológicas que en la actualidad juegan un importante papel en el proceso enseñanza aprendizaje.

Sin embargo, al contar con las TD como herramientas académicas que incentiven a los estudiantes en su formación, estas a su vez abren una ventana de posibilidades para afrontar de 
mejor manera estas necesidades educativas. Es de gran importancia pensar en los beneficios que conlleva el uso de las TD en la adquisición de conocimientos para los estudiantes, esta realidad se evidencia como una problemática por dos condiciones específicas en Ecuador, la primera es el escaso acceso a la tecnología que tiene la población estudiantil en general y se enfatiza en el uso minoritario de las TIC en la educación (MINEDUC, 2013); la segunda es la falta de afluencia dentro de los espacios de capacitación por parte del personal docente y administrativo acerca del uso de las TIC dentro de los nuevos modelos educativos.

Para el presente trabajo de investigación se pretende conocer el nivel de competencias tecnológicas , pedagógicas y de contenido del profesorado universitario a través del modelo TPACK, de esta manera surge el cuestionamiento de conocer ¿Cuál es el nivel de competencias docentes en la utilización de las TD, en el proceso enseñanza/aprendizaje en el profesorado universitario?, al dar respuesta a esta interrogante se podrá ofrecer un marco claro de desarrollo profesional docente que sirva como referente para orientar las decisiones sobre formación y actualización del profesorado.

\section{Marco Teórico}

\section{Competencias docentes en el manejo de las TIC}

Se considera la competencia digital como un derecho del ser humano y se entiende como un concepto que va más allá de la competencia técnica, en la cual se asume la inclusión de áreas de competencia como: gestión de la información; colaboración; capacidad de comunicación y de compartir información; creación de contenidos y conocimiento; ética y responsabilidad; evaluación y solución de problemas; y finalmente, la técnica (Ferrari, 2013). Concebir la integración de la competencia digital en el desarrollo profesional docente es el reto de la educación moderna por lo que se abren "nuevos paradigmas de investigación en el campo de la educación y en el paradigma de la investigación de los procesos de enseñanza/aprendizaje" (Gewerc, Pernas, y Varela, 2013).

Es así que se pretende que los docentes, en su trabajo cotidiano, relacionen estos ámbitos del conocimiento. Pues en definitiva no es suficiente contar con un elevado conocimiento en la cátedra sino también de emplear la metodología adecuada para la enseñanza. "En definitiva, parece fiable pensar que la igualdad de estas partes es la que garantiza la integración de las TD en la docencia” (Ramírez, 2004).

Es indudable que los profesores de hoy en día deben desarrollar una serie de competencias para la docencia las cuales les permitan hacer frente a los nuevos retos educativos y sociales, destacando entre ellas la competencia digital (Esteve, 2015). De este modo, será necesario que los docentes se desenvuelvan en el mundo digital, en la creación y distribución de contenidos y recursos, en diferentes contextos y con diferentes dispositivos (Salinas, De Benito y Lizana, 2014)

En definitiva, estas nuevas tecnologías se están incorporando en la educación, y esto ha traído cambios importantes en los procesos enseñanza/aprendizaje en todos los niveles de la 
educación. También ha permitido que los docentes no se queden atrás y empiecen a utilizar las herramientas tecnológicas actuales, aunque es importante recalcar que existe aún resistencia a integrarlas en el aula, y más bien las utilizan como apoyo a sus actividades docentes más que como un apoyo a los aprendizajes de los estudiantes.

Las TIC "son generadoras de nuevos conocimientos, de procesos de innovación dinámicos y eficaces hechos que abren nuevas posturas y nuevas formas de concebir la educación y el mundo, una realidad inocultable donde la información circunda por todas partes y donde las formas de procesarla son cada vez más novedosas" (Cejas, Navío, y Barroso, 2016).

"Para comprender las relaciones existentes entre el conocimiento experto que posee un profesor en su asignatura con su conocimiento pedagógico mediados por sus conocimientos tecnológicos, el modelo TPACK" (Cejas, Navío, y Barroso, 2016), permite la integración de estos elementos en el proceso enseñanza aprendizaje. "Sin duda alguna, en la actualidad urge integrar la tecnología en el ámbito educativo, pero no de cualquier modo es así que el modelo TPACK viene a reiterar la relación entre estos elementos que el docente debe saber llevar al aula". (Centros XXI, 2013).

\section{Modelo TPACK}

Un modelo que ha adquirido cierta trascendencia en los últimos años, es el TPACK (Conocimiento Pedagógico Tecnológico del Contenido) se conceptualiza como un modelo que sirve a los docentes para diseñar sus clases mediante el uso de TD. Este modelo propone al docente la correlación del conocimiento tecnológico, pedagógico y de contenido (Koehler y Mishra, 2009).

La propuesta sugiere que la capacitación de los docentes en TD, debe pasar por la adquisición de tres tipos de conocimientos básicos: tecnológicos (TK), pedagógicos (PK) y de contenidos o disciplinar $(\mathrm{CK})$, indicándose a través del modelo que lo importante no es formar a los docentes en estos conocimientos de forma aislada, sino en combinación: PCK: Conocimiento Pedagógico del Contenido; TCK: Conocimiento de la utilización de las tecnologías; TPK: Conocimiento pedagógico tecnológico; y TPACK: Conocimiento Tecnológico, pedagógico y de contenido (figura 1). 


\section{Figura 1}

\section{Conceptualización del TPACK como modelo}

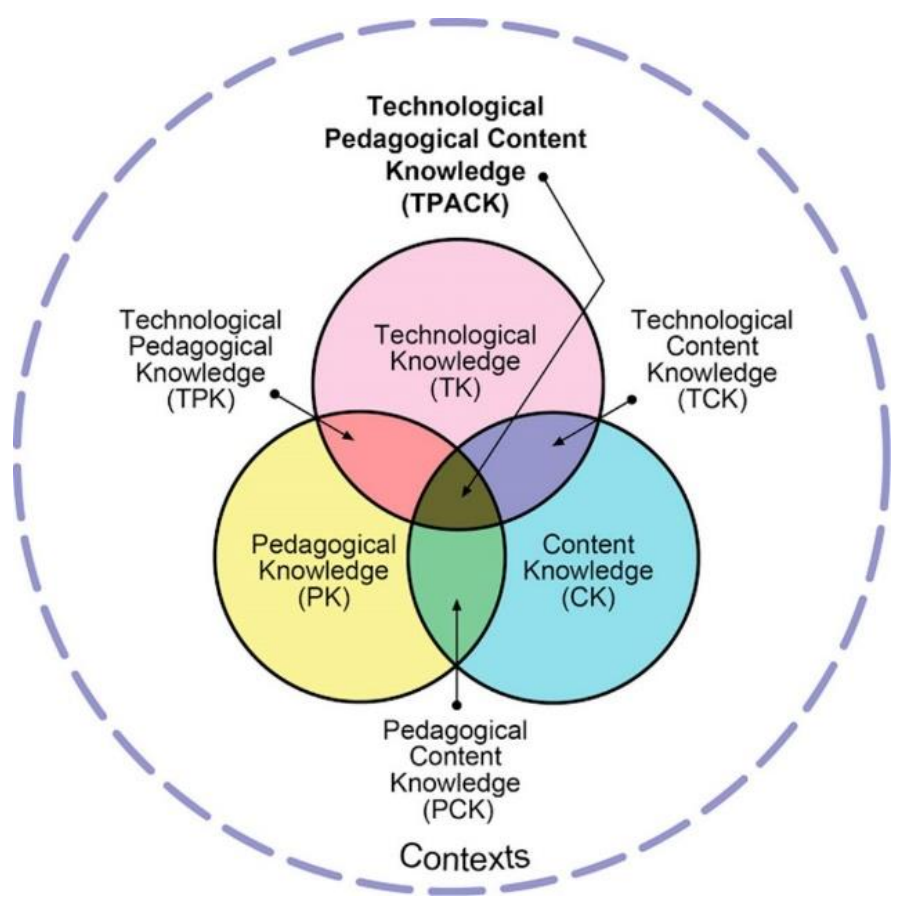

En este contexto se define las tres principales competencias tal como la competencia disciplinar (CK) que menos atención se le ha prestado en la elaboración del modelo, el motivo puede ser porque se supone que todo profesor ha sido formado previamente en la disciplina que imparte; competencias pedagógicas (PK), este conocimiento es independiente de la asignatura que imparte, pues todo profesor universitario debe ser competente en la planificación de la formación, en el diseño de escenarios de aprendizaje, en la orientación del alumno en su propio aprendizaje y en la evaluación. Además, sobrepasando las funciones propias del proceso de enseñanza-aprendizaje, debe contribuir a la mejora de la docencia y participar en la dinámica de la propia institución en la que trabaja, convirtiéndose en un potente agente de cambio; y las competencias tecnológicas (TK), que su conocimiento y uso de la tecnología debe ser dinámica a la par con la constante evolución de nuevas tecnologías y su diversidad en los amplios usos que se pueda dar, estas competencias antes descritas forman parte del esquema principal y de las que se desprende las dimensiones emergentes como los (PCK), (TCK), (TPK) Y (TPACK). A continuación, se presenta una tabla de ejemplos de la aplicación del modelo TPACK. 
Tabla 1

Ejemplo dimensiones principales y emergentes del modelo TPACK

\section{Dimensiones TPACK}

TK. Competencias

Tecnológicas (Technological

Knowledge)

PK. Competencias

Pedagógicas (Pedagogical

Knowledge)

CK. Competencias

Disciplinares (Content

Knowledge)

TPK. Competencias

Tecnológicas Pedagógicas.

(Technological Pedagogical

Knowledge)

\section{Descripción}

Conocimientos sobre

capacidades y aplicaciones tecnológicas

Competencias pedagógicas en general.

Ejemplos

Usar Symbaloo. Crear un blog. Conectar un dispositivo móvil en el ordenador.

Dinamizar grupos de alumnos en la resolución de problemas. Evaluar por competencias.

Conocer la ecuación de Competencias sobre la materia en la que es experto.

Drake. Formular la sacarosa a partir de glucosa y fructosa.

Competencias que incluyen aspectos tecnológicos y pedagógicos.
Conocer cómo Prezi puede usarse para que los alumnos trabajen colaborativamente en una presentación oral en clase.
TCK. Competencias

Tecnológicas del Contenido. (Technological Content Knowledge)
Conocimientos sobre cómo la tecnología puede utilizarse para representar la materia a utilizar y desarrollar la competencia disciplinar.

Conocimientos pedagógicos que faciliten que los alumnos adquieran determinadas habilidades o contenidos.

Conocimientos sobre cómo usar la tecnología más adecuada en un marco pedagógico para la impartición de determinada materia.
Utilizar la realidad virtual para generar modelos físicos teóricos. Diseñar una animación que refleje el ciclo de los ácidos tricarboxílicos.

Crear una guía didáctica ilustrada para que los alumnos sepan cómo diseccionar una rana

Dinamizar un grupo de alumnos para que trabajen colaborativamente online en el diseño tridimensional del sistema solar (Technological Pedag
Content Knowledge)

Nota: La información refleja un ejemplo del modelo TPACK, tabla extraída de Cejas León et al. (2016). Las Competencias Del Profesorado Universitario Desde El Modelo TPACK (Conocimiento Tecnológico Y Pedagógico Del Contenido) 


\section{Metodología}

El presente estudio se enmarca en una investigación no experimental o expost-facto ya que se encuentra en una situación real natural en la que se observan las variables sin ser manipuladas deliberadamente, tiene un enfoque cuantitativa descriptiva y transversal debido a que se realizó la recolección de datos mediante la aplicación de un encuesta para integrar una valoración veraz de la información, que lograra determinar las competencias digitales que el profesorado universitario posee, a su vez es transversal dado que el estudio se realizó en un solo periodo de tiempo.

La muestra fue de 238 docentes universitarios quienes se les aplico la técnica de recolección de datos mediante la encuesta y como instrumento el cuestionario TPACK modificado de Schmidt et al ( 2009), los cuales están constituidas de 7 dimensiones distribuidas en 32 preguntas, con valoraciones de 1: Deficiente 2: Bajo 3: Aceptable 4: Bueno 5 Excelente, es así que las preguntas están organizadas de la siguiente manera:

- Información General (4 ítems).

- Conocimiento tecnológico (TK) (6 ítems).

- Conocimiento del contenido (CK) (3 ítems).

- Conocimiento pedagógico (PK) (7 ítems).

- Conocimiento pedagógico del contenido (PCK) (1 ítems).

- Conocimiento tecnológico del contenido (TCK) (1 ítems).

- Conocimiento tecnológico pedagógico (TPK) (5 ítems).

- Conocimiento tecnológico pedagógico del contenido (TPACK) (5 ítems).

Con el propósito de realizar la investigación, se estructuraron los instrumentos de recolección de datos, para lo cual se definieron una encuesta piloto basada en el Cuestionario TPACK con un número de 10 encuestas a docentes, con el propósito de definir si sus enunciados son comprensibles al encuestado y obtener datos que permitan realizar un examen de confiabilidad. Con la información obtenida de las encuestas piloto se procedió a realizar el análisis de CRONBACH con el objeto de determinar si las encuestas son confiables.

\section{Tabla 2}

Alfa de Cronbach

Estadísticas de fiabilidad

\begin{tabular}{|c|c|c|}
\hline & $\begin{array}{c}\text { Alfa de } \\
\text { Cronbach } \\
\text { basada en } \\
\text { elementos } \\
\text { Alfa de } \\
\text { estandarizad } \\
\text { os }\end{array}$ & $\begin{array}{c}\mathrm{N} \text { de } \\
\text { elementos }\end{array}$ \\
\hline, 733 & \multicolumn{2}{|c|}{32} \\
\hline
\end{tabular}


Como se puede apreciar en la tabla 2, el valor de Alfa de Cronbach es de 0.733, que representa la fiabilidad del tipo de consistencia interna de una escala, que permitió evaluar la magnitud en que los ítems del instrumento están correlacionados, cuyo valor resultante es aceptable, lo cual permitió proceder a aplicar la encuesta a toda la muestra estadística.

Los datos fueron procesados por medio del sistema estadístico SPSS V. 23 a fin de obtener la data que ayudara a realizar los análisis descriptivos que nos permitan establecer los resultados de la investigación.

\section{Resultados y Discusión}

\section{Exposición de Resultados de encuesta aplicada a los docentes}

En este apartado se muestran los resultados obtenidos en la investigación tras aplicar diferentes pruebas estadísticas. Así, respecto a las puntuaciones alcanzadas por los docentes en los diferentes ítems del cuestionario, se calcularon distribuciones con respecto a la media y la desviación típica que se presenta en la tabla 3.

\section{Tabla 3}

Puntuaciones TPACK

\begin{tabular}{lcccc}
\hline \multicolumn{1}{c}{ Dimensiones } & Mínimo & Máximo & Media & $\begin{array}{c}\text { Desviación } \\
\text { estándar }\end{array}$ \\
\hline C. tecnológico (TK) & 2 & 5 & 3,9516 & 0.8925 \\
C. contenido (CK) & 3 & 5 & 3,6839 & 0.49357 \\
C. pedagógico (PK) & 3 & 5 & 3,6132 & 0.52403 \\
C. pedagógico del contenido (PCK) & 3 & 5 & 3,4138 & 0.52805 \\
C. tecnológico del contenido (TCK) & 1 & 5 & 3,1724 & 0.93917 \\
C. tecnológico pedagógicos (TPK) & 3 & 5 & 3,3276 & 0.65344 \\
C. tecnológico pedagógico del contenido & 2 & 5 & 3,2310 & 0.761862 \\
(TPACK) & & & & \\
\hline
\end{tabular}

Nota: La tabla muestra los valores obtenidos en cada una de las dimensiones del modelo TPACK por parte del profesorado.

Como se puede apreciar en la tabla 3 se muestran los resultados generales obtenidos tras aplicar la encuesta al profesorado universitario y analizado desde las diferentes pruebas estadísticas. 


\section{Figura 2}

\section{Puntuaciones medias subescalas del TPACK}

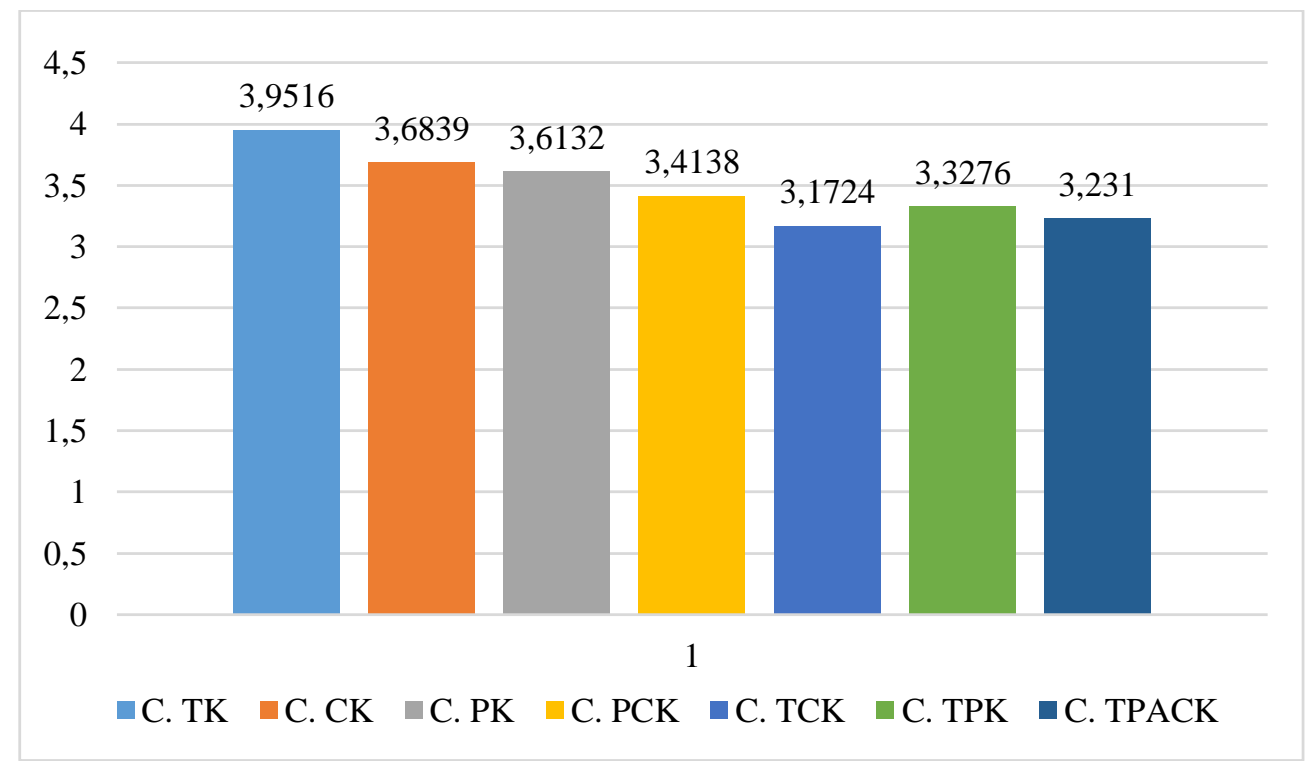

Nota: El gráfico representa la media obtenida de la aplicación del modelo TPACK al profesorado.

Se puede observar que las valoraciones alcanzadas por parte del profesorado en las tres principales variables, su puntuación media es superior a 3.6, y se manifiestan en desviaciones estándar muy homogéneas, esto muestra que los profesores encuestados tienen una alta autopercepción de las variables principales (TK, CK, PK) capturados en el modelo TPACK. Al mismo tiempo llama la atención que los conocimientos tecnológicos que el profesorado posee son altos seguido por el conocimiento disciplinar y pedagógico, estos resultados son similares a los obtenidos en investigaciones realizadas por (Cabero-Almenara, 2018)(Cabero et al., 2017; Koh y Chai, 2014), de manera análoga mencionan Cando et al. (2018) "que la actual formación de los docentes en TIC para la práctica pedagógica es deficiente.

Por otro lado, se evidencia una competencia menor en la conjugación de dos o más escalas, básicamente en la competencia tecnológica de contenido, tecnológica pedagógica, tecnológico pedagógico del contenido y pedagógica del contenido, esta información demuestra claramente que el profesorado universitario tiene dificultades en la capacidad de combinar recursos didácticos y métodos pedagógicos apropiados para el proceso enseñanza aprendizaje dando una imagen de divergencia entre las competencias.

La investigación tiene similitud a los resultados obtenidos por el equipo de investigación de Cabero $(2014,2015)$ quienes afirman que las diferentes funciones tecnológicas ayudan, pero no bastan, para el desarrollo del TPACK de un profesor. De hecho, muchos profesores con CK y PK elevados, encuentran dificultades para encontrar la tecnología adecuada en su quehacer docente. 
En relación con los resultados estos dan a conocer que los recursos tecnológicos que el profesorado universitario posee no logran integrar significativamente las diferentes competencias del TPACK.

\section{Conclusiones}

El estudio invita a conocer cuál es el nuevo rol del profesorado universitario en miras a la gran avalancha de tecnología que se puede aplicar en el proceso enseñanza aprendizaje, para lo cual es necesario recalcar que para lograr que la tecnología en asociación con la pedagogía y el conocimiento de la asignatura logren eficientemente un entorno adecuado para el proceso educativo, el profesorado tendrá que seguir un proceso complejo con la aplicación de distintas variables, cabe mencionar que sin duda alguna solamente la tecnología no será la solución a los grandes problemas que la educación enfrenta en la actualidad.

El modelo TPACK brinda las pautas para una fundamentación teórica de las investigaciones en Tecnología Educativa e inspira la elaboración de instrumentos de diagnóstico, evaluación y seguimiento de integración de las TIC en la práctica docente, partiendo de esta base el estudio logra analizar las competencias digitales al profesorado universitario mediadas por el modelo TPACK, los resultados obtenidos son poco alentadores, sin embargo su competencia docente es aceptable teniendo grandes desafíos al aplicar la tecnología en sinergia con la pedagogía y la disciplina, sin embargo si el profesorado lograra conjugar satisfactoriamente estas dimensiones se podrá asegurar la calidad de la enseñanza y aprendizaje mediada por la tecnología

Se puede mencionar que las competencias que los docentes poseen están en constante evolución, es así que entre las competencias tecnológicas, pedagógicas y disciplinares de manera independiente logran alcanzar competencias altas en el profesorado, no siendo así las competencias emergentes del TPACK donde se manifiesta una disminución en la integración de las mismas y este echo alcanzará a impactar significativamente en el proceso enseñanza aprendizaje, desde otro punto de vista estos resultados no solo nos permite conocer la situación actual del profesorado en la integración de las TD, si no que en consecuencia brinda una visión de la formación de los estudiantes con entorno digitales.

El dominio, uso e innovación de la competencia digital docente es imprescindible para favorecer cambios en la educación superior buscando la adquisición de aprendizajes duraderos para construir conocimiento y generar transformaciones significativas en la educación superior, sin embargó el hecho de implementar la tecnología para la enseñanza y aprendizaje es una tarea verdaderamente compleja, cuyo éxito dependerá de un numero de variables a aplicarse, de las cuales solo una es la tecnología.

Finalmente, es importante señalar que el profesorado universitario a más de su función docente, se involucra en otras funciones sustantivas universitarias como es la investigación, vinculación con la sociedad y la gestión, esto implica también elaborar nuevas competencias para cada una de estas funciones y cómo las tecnologías pueden ayudar en su desarrollo. 


\section{Referencias bibliográficas}

Acevedo, J., Martínez, C., \& Cruz, B. (2014). El uso de las TIC en el aula: un análisis en términos de efectividad y eficacia. Buenos Aires.

Alarcón, A. (2017). Tecnologías De La Información Y La Comunicación. Obtenido de https://es.calameo.com/books/005009326b693924045a3

Almenara, J. C., Díaz, V. M., \& Garrido, C. C. (15 de Julio de 2015). Biblioteca Universidad de Córdoba. Obtenido de Validación de la aplicación del modelo TPACK para la formación del profesorado en TIC: https://helvia.uco.es/bitstream/handle/10396/17286/\%40tic\%202015.pdf?sequence=1\&isA llo wed=y

Arias, F. (2012). Proyecto de Investigación Científica. Introducción a la metodología científica. 6a Edición. Caracas: Episteme.

Arista, J. (2014). Tecnologías de la Información y la Comunicación (TIC) aplicadas a la docencia. UAEH; Universidad Autónoma del Estado de Hidalgo. Asamblea, N. (2008). Constitución Política del Ecuador. Quito.

Bernal, D. (2016). Arte y pedagogía. Obtenido de http://files.educacionpopular8.webnode.es/200000005-a273ca4682/arte_y_pedagogia.pdf

Bernardi, G. A. (2018). Las TIC como recurso educativo / su inclusión en las aulas universitarias para enriquecer propósitos y contenidos. RIIDA, 2-27. Obtenido de https://ridaa.unq.edu.ar/bitstream/handle/20.500.11807/852/TFI_2018_bernardi_010.pdf

?sequ ence $=1 \&$ isAllowed $=\mathrm{y}$

Bisquerra, R. (2004). Metodología de la Investigación Educativa. Madrid: La Muralla.

Cabero, J. (22 de junio de 2015). Biblioteca Universidad de Córdoba. Obtenido de Validación de la aplicación del modelo TPACK para la formación del profesorado en TIC: https://idus.us.es/xmlui/handle/11441/32293

Cala, R. (2018). The Impact of the Use of Interactive Digital Screens (IDS) in the Teaching Learning Process. Scielo, 1-10. Obtenido de https://scielo.conicyt.cl/scielo.php?script=sci_arttext\&pid=S0718$07642018000500061 \&$ lang=en

Cejas, R., Navío, A., \& Barroso, J. (2016). Las competencias del profesorado universitario desde el modelo TPACK (Conocimiento Tecnológico y Pedagógico Del Contenido). Píxel-Bit. Revista de Medios y Educación, 49., 105-119. Obtenido de https://idus.us.es/xmlui/bitstream/handle/11441/44210/Las_competencias_del_profesora do_universitario_desde_el_modelo_TPACK_conocimiento.pdf?sequence=1\&isAllowed $=\mathrm{y}$

Centros XXI. (29 de enero de 2013). Educar en el siglo XXI. Un lugar para reflexión sobre aprendizaje, competencias, Oducación. Obtenido de http://centrosxxi.blogspot.com/2013/01/que-es-el-modelo-tpack_29.html

Chocó, A. (2015). Educación Pedagógica y TIC. Un Trinomio imperioso en la nueva perspectiva social. Obtenido de https://arbercho.jimdo.com/pedagog\%C3\%ADa-y-tic/

Cochrane, T. A. (2014). Developing a mobile social media framework for creative pedagogies. Proceedings of the 10th International Conference on Mobile Learning, 207-214.

Constituyente, A. (2008). Constitución Politica del Ecuador. Quito. 
Del Toro, V. (2015). Introducción de las TIC en el proceso enseñanza-aprendizaje en el sector Salud y en Universidad Ciencias Médicas Guantánamo. Guantánamo.

Echeverria, A. (2014). Universidad de Costa Rica. Obtenido de https://revistas.ucr.ac.cr/index.php/aie/article/view/16131

Freire, P. (28 de marzo de 2017). La pedagogía liberadora vs pedagogía cognitiva. La Hora, págs. $\quad$ https://lahora.com.ec/noticia/1102043210/la-pedagogc3ada-liberadora-vspedagogc3ada-cognitiva.

García, R., \& González, N. (2005). El aprendizaje cooperativo como alternativa metodológica en la formación universitaria. Comunicación y Pedagogía.

Gelves, R. (2010). Uso de Simuladores como Recurso Digital para Transferencia de Conocimiento. Innovación Educativa, $\quad 1-16 . \quad$ Obtenido de http://www.udgvirtual.udg.mx/apertura/index.php/apertura/article/view/22

Gewerc, A., Pernas, E., \& Varela, J. (2013). Conocimiento tecnológico-didáctico del contenido en la enseñanza de Ingeniería Informática: un estudio de caso colaborativo con la perspectiva del docente y los investigadores. Revista de Docencia Universitaria, 2-8.

Gonzáles, F. (1997). Epistemología Cualitativa y Subjetividad. La Habana: Pueblo y Educación. Google. (16 de febrero de 2013). Google sites. Obtenido de Universidad Autónoma de Zacatecas: https://sites.google.com/site/etiekllp/clasificacion-de-medios-de-ensenanzaaprendizaje/medios-manipulativos

Googlesites. (05 de Abril de 2018). LAS TIC EN EDUCACION. El TPACK: un marco teórico conceptual para integrar las tecnologías a la educación. Obtenido de https://sites.google.com/site/finalinformaticayprogramacion/el-tpack-un-marco-teoricoconceptual-para-integrar-las-tecnologias-a-la-educacion

Hermosa, P. (2015). Influencia de las tecnologías de información y comunicación (TIC) en el proceso enseñanza-aprendizaje: una mejora de las competencias digitales. Bogotá: Rev. Cient’. Gen. José María Córdova.

Hernández, Fernández, \& Batista. (2003). Metodología de Investigación.

Hockly, N. (2012). Mobile learning. Elt Journal, 64.

Jiménez, L. (31 de marzo de 2017). Tesis Doctorals de Xarxa. Obtenido de Universitat Ramon Llull: https://www.tdx.cat/handle/10803/402469

Jimmy, R. (2006). TIC. Obtenido de https://ddd.uab.cat/pub/dim/16993748n8/16993748n8a6.pdf Levin, D., \& Tsybulsky, I. (2015). New Perspectives in Science Education. Obtenido de SAMR Framework for Study Technology Integration in Science Education: http://m.tau.ac.il/ ilia1/perspectives-florence.pdf

Malbernat, R. (2011). Repositorio institucional de la UNPL. Obtenido de http://sedici.unlp.edu.ar/handle/10915/18307

Mares, L. (2012). Tablets en Educación / Oportunidades y Desafíos en Políticas Uno a Uno. RELPE, 4-7. Obtenido de file:///C:/Users/HP/Downloads/Tabletseneducacion.pdf

MINEDUC. (10 de abril de 2013). Aula Digital Móvil. Obtenido de https://educacion.gob.ec

Mouza, C., Karchmmer, R., \& Yilmaz, S. (2014). Investigating the impact of an integrated approach to the development of preservice teachers' technological pedagogical content knowledge (TPACK). Computers \& Education, 13-18.

Nessipbayeva, O. (2015). Pre-Service and In-Service Teacher Training. Obtenido de https://files.eric.ed.gov/fulltext/ED567059.pdf 
Pérez, A. R. (27 de marzo de 2018). Actividades de Aula en el Ámbito de la Tecnología con dispositivos de Impresión. Obtenido de http://uvadoc.uva.es/bitstream/10324/33338/1/TFM- G957.pdf

Pérez, M. (2008). Competencias adquiridas por los futuros docentes desde la formación inicial. Revista de educación, 343.

Posada, F. (8 de mayo de 2013). canal TIC. Obtenido de https://canaltic.com/blog/?p=1677

Puentedura, R. (2006). Hippasus. Obtenido de Hippasus: http://hippasus.com/resources/tte/puentedura_tte.pdf

Ramírez, M. (2004). Las tecnologías de la información y la comunicación aplicadas a la docencia universitaria en Epigrafía y Numismática: presente y futuro. Obtenido de http://www.cervantesvirtual.com/obra-visor/las-tecnologias-de-la-informacion-y-lacomunicacion-aplicadas-a-la-docencia-universitaria-en-epigrafia-y-numismaticapresente-y-futuro--0/html/0017ccba-82b2-11df-acc7-002185ce6064_3.html

Solanes, A., \& Núñez, R. (2008). Elaboración de un cuestionario para la evaluación de competencias genéricas en estudiantes universitarios. Apuntes de Psicología, 35-49.

Svetlichich, M. E. (2015). Nuevas Herramientas Tecnológicas en la Educación Superior. XXXI Conferencia Interamericana de Contabilidad (págs.). Punta Cana / Republica Dominicana: SEDICI, (págs. 93-149). Punta Cana.

Tejada, J. (2009). Competencias docentes. Revista de Currículum y Formación de Profesorado, 15.

Torres, C. (11 de Noviembre de 2013). Uso de las TIC e internet dentro y fuera del aula. Obtenido de http://www.udgvirtual.udg.mx/apertura/index.php/apertura/article/view/381/319

UNESCO, O. d. (20 de 08 de 2016). UNESCO. Obtenido de http://www.unesco.org/new/es/santiago/education/teachers/teachers-icts/ 


\section{Anexo 1}

\section{Encuesta aplicada al Profesorado Universitario}

\section{Encuesta dirigida al profesorado universitario}

Objetivo: recabar información acerca de las competencias docentes en tecnologías digitales

Información general:

\section{CUESTIONARIO}
a) Género: Masculino ( )
Femenino ( )

b) Edad

c) Seleccione de la siguiente tabla sus años de experiencia docente, colocando una (X) al frente del enunciado que corresponda.

\begin{tabular}{|l|l|l|}
\hline 1 & Menos de 1 año & \\
\hline 2 & De 1 a 3 años & \\
\hline 3 & De 3 a 5 años & \\
\hline 4 & De 5 a 7 años & \\
\hline 5 & De 7 a 10 años & \\
\hline 6 & De 10 a 15 años & \\
\hline 7 & De 15 a 20 años & \\
\hline 8 & Más de 20 años & \\
\hline
\end{tabular}

d) En el siguiente recuadro escriba en nombre de la cátedra con mayor carga horaria que actualmente tiene.

\begin{tabular}{|l|l|}
\hline 1 & \\
\hline 2 & \\
\hline 3 & \\
\hline 4 & \\
\hline 5 & \\
\hline
\end{tabular}


A continuación, se insertan preguntas del cuestionario Tpack, orientado a conocer las competencias docentes en el uso de las TICs. Lea detenidamente cada una de las preguntas y responda colocando una (x) en el número que corresponda según la escala designada.

1. En cuanto a su conocimiento tecnológico responda los siguientes enunciados en la escala del 1 al 5. Siendo: 1 Deficiente, 2 Bajo, 3 Aceptable, 4 Bueno, 5 Excelente.

\begin{tabular}{|c|c|c|c|c|c|c|}
\hline \multicolumn{7}{|c|}{ 1. Conocimiento tecnológico (TK) } \\
\hline & 1: Deficiente 2: Bajo 3: Aceptable 4: Bueno 5 Excelente & 1 & 2 & 3 & 4 & 5 \\
\hline TK 1.1 & Sé resolver mis problemas técnicos. & & & & & \\
\hline TK 1.2 & Asimilo conocimientos tecnológicos fácilmente. & & & & & \\
\hline TK 1.3 & Me mantengo al día de las nuevas tecnologías importantes & & & & & \\
\hline TK 1.4 & A menudo juego y hago pruebas con la tecnología & & & & & \\
\hline TK 1.5 & Conozco muchas tecnologías diferentes. & & & & & \\
\hline TK 1.6 & $\begin{array}{l}\text { He tenido oportunidades suficientes de trabajar con diferentes } \\
\text { tecnologías }\end{array}$ & & & & & \\
\hline
\end{tabular}

2. En cuanto al conocimiento del contenido de la cátedra que usted imparte responda los siguientes enunciados en la escala del 1 al 5. Siendo: 1 Deficiente, 2 Bajo, 3 Aceptable, 4 Bueno, 5 Excelente.

\begin{tabular}{|c|c|c|c|c|c|c|}
\hline \multicolumn{7}{|c|}{ 2. Conocimiento del contenido $(\mathbf{C K})$} \\
\hline & 1: Deficiente 2: Bajo 3: Aceptable 4: Bueno 5 Excelente & 1 & 2 & 3 & 4 & 5 \\
\hline CK 2.1 & Tengo suficientes conocimientos sobre la cátedra que imparto & & & & & \\
\hline CK 2.2 & Sé aplicar un modo de pensamiento según la cátedra que imparto & & & & & \\
\hline CK 2.3 & $\begin{array}{l}\text { Tengo varios métodos y estrategias para desarrollar mi conocimiento } \\
\text { sobre la cátedra que imparto }\end{array}$ & & & & & \\
\hline
\end{tabular}

3. En cuanto al conocimiento pedagógico responda los siguientes enunciados en la escala del 1 al 5. Siendo: 1 Deficiente, 2 Bajo, 3 Aceptable, 4 Bueno, 5 Excelente.

\begin{tabular}{|c|c|c|c|c|c|c|}
\hline \multicolumn{7}{|c|}{ 3. Conocimiento Pedagógico (PK) } \\
\hline & 1: Deficiente 2: Bajo 3: Aceptable 4: Bueno 5 Excelente & 1 & 2 & 3 & 4 & 5 \\
\hline PK 3.1 & Sé cómo evaluar el rendimiento del alumnado en el aula. & & & & & \\
\hline PK 3.2 & $\begin{array}{l}\text { Sé adaptar mi docencia a lo que el alumnado entiende o no entiende } \\
\text { en cada momento }\end{array}$ & & & & & \\
\hline
\end{tabular}




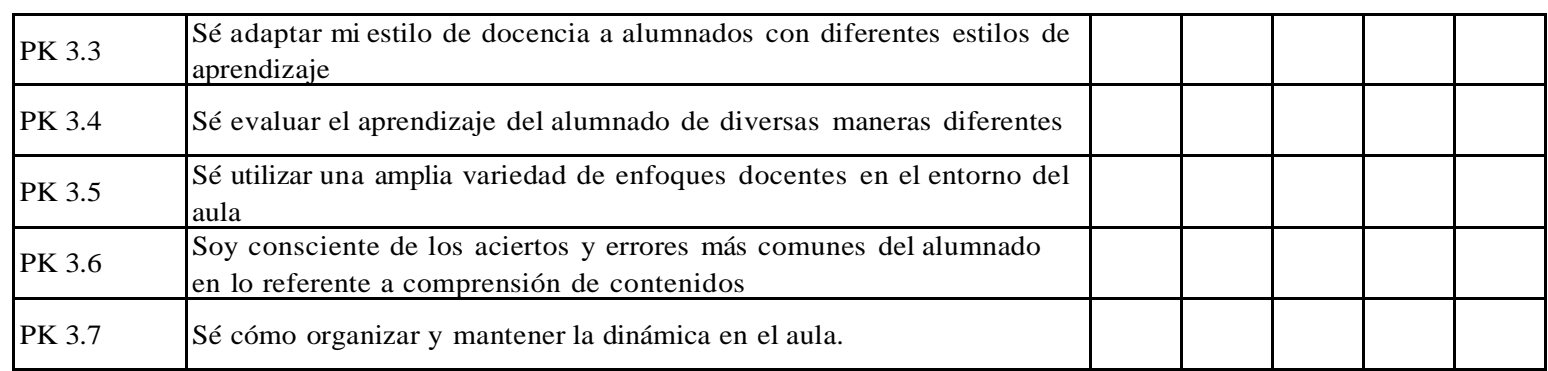

4. En cuanto al conocimiento pedagógico del contenido responda los siguientes enunciados en la escala del 1 al 5. Siendo: 1 Deficiente, 2 Bajo, 3 Aceptable, 4 Bueno, 5 Excelente.

\section{Conocimiento Pedagógico del Contenido (PCK)}

\begin{tabular}{|c|c|c|c|c|c|c|}
\hline & 1: Deficiente 2: Bajo 3: Aceptable 4: Bueno 5 Excelente & 1 & 2 & 3 & 4 & 5 \\
\hline PCK 4.1 & $\begin{array}{l}\text { Puedo seleccionar enfoques docentes de manera eficaz para guiar el } \\
\text { pensamiento y el aprendizaje del alumnado en la cátedra que imparto }\end{array}$ & & & & & \\
\hline
\end{tabular}

5. En cuanto al conocimiento tecnológico del contenido responda los siguientes enunciados en la escala del 1 al 5. Siendo: 1 Deficiente, 2 Bajo, 3 Aceptable, 4 Bueno, 5 Excelente.

\section{Conocimiento tecnológico del contenido (TCK)}

\begin{tabular}{|c|c|c|c|c|c|c|}
\hline & 1: Deficiente 2: Bajo 3: Aceptable 4: Bueno 5 Excelente & 1 & 2 & 3 & 4 & 5 \\
\hline TCK 5.1 & $\begin{array}{l}\text { Conozco tecnologías que puedo usar para comprender y elaborar } \\
\text { contenidos sobre la cátedra que imparto }\end{array}$ & & & & & \\
\hline
\end{tabular}

6. En cuanto al conocimiento tecnológico - pedagógico responda los siguientes enunciados en la escala del 1 al 5. Siendo: 1 Deficiente, 2 Bajo, 3 Aceptable, 4 Bueno, 5 Excelente.

\begin{tabular}{|c|c|c|c|c|c|c|}
\hline \multicolumn{7}{|c|}{ Conocimiento tecnológico pedagógico (TPK) } \\
\hline & 1: Deficiente 2: Bajo 3: Aceptable 4: Bueno 5 Excelente & 1 & 2 & 3 & 4 & 5 \\
\hline TPK 6.1 & $\begin{array}{l}\text { Sé seleccionar tecnologías que mejoran los enfoques docentes para } \\
\text { una lección. }\end{array}$ & & & & & \\
\hline TPK 6.2 & $\begin{array}{l}\text { Sé seleccionar tecnologías que mejoran el aprendizaje del alumnado } \\
\text { en una lección }\end{array}$ & & & & & \\
\hline TPK 6.3 & $\begin{array}{l}\text { Mi formación como docente me ha hecho reflexionar más } \\
\text { detenidamente sobre la forma en que la tecnología puede influir en los }\end{array}$ & & & & & \\
\hline TPK 6.4 & $\begin{array}{l}\text { Adopto un pensamiento crítico sobre la forma de utilizar la tecnología } \\
\text { z el aula. }\end{array}$ & & & & & \\
\hline TPK 6.5 & $\begin{array}{l}\text { Puedo adaptar el uso de las tecnologías sobre las cuales estoy } \\
\text { aprendiendo a diferentes actividades docentes. }\end{array}$ & & & & & \\
\hline
\end{tabular}

7. En cuanto al conocimiento tecnológico - pedagógico del contenido de la cátedra que usted imparte, responda los siguientes enunciados en la escala del 1 al 5. Siendo: 1 Deficiente, 2 Bajo, 3 Aceptable, 4 Bueno, 5 Excelente. 


\section{Conocimiento tecnológico pedagógico del contenido (TPACK)}

\begin{tabular}{|c|c|c|c|c|c|c|}
\hline \multicolumn{2}{|r|}{ 1: Deficiente 2: Bajo 3: Aceptable 4: Bueno 5 Excelente } & 1 & 2 & 3 & 4 & 5 \\
\hline TPACK 7.1 & $\begin{array}{l}\text { Puedo impartir lecciones que combinan adecuadamente cátedra, } \\
\text { tecnologías y enfoques docentes. }\end{array}$ & & & & & \\
\hline TPACK 7.2 & $\begin{array}{l}\text { Sé seleccionar tecnologías para usar en el aula que mejoran los } \\
\text { contenidos que imparto, la forma de impartirlos y lo que aprende el } \\
\text { alumnado. }\end{array}$ & & & & & \\
\hline TPACK 7.3 & $\begin{array}{l}\text { Sé usar en mis materiales docentes para el aula estrategias que } \\
\text { combinan contenidos, tecnologías y enfoques docentes sobre los } \\
\text { cuales he aprendido. }\end{array}$ & & & & & \\
\hline TPACK 7.4 & $\begin{array}{l}\text { Puedo guiar y ayudar a otras personas a coordinar el uso de } \\
\text { contenidos, tecnologías y enfoques docentes en mi centro docente } \\
\text { y/o región administrativa. }\end{array}$ & & & & & \\
\hline TPACK 7.5 & $\begin{array}{l}\text { Puedo seleccionar tecnologías que mejoran el contenido de las } \\
\text { lecciones. }\end{array}$ & & & & & \\
\hline
\end{tabular}

¡iMUCHAS GRACIAS POR SU GENTIL COLABORACIÓN! 